\title{
Two-dimensional photon echoes reveal non-Markovian energy transfer in an excitonic dimer
}

\author{
Hong-Guang Duan, ${ }^{1,2,3}$ Moritz Frey, ${ }^{1,4}$ Michael Thorwart,, ${ }^{1,2}$ and Peter Nalbach ${ }^{5}$ \\ ${ }^{1}$ I. Institut für Theoretische Physik, Universität Hamburg, Jungiusstraße 9, 20355 Hamburg, Germany \\ ${ }^{2}$ The Hamburg Centre for Ultrafast Imaging, Luruper Chaussee 149, 22761 Hamburg, Germany \\ ${ }^{3}$ Max Planck Institute for the Structure and Dynamics of Matter, Luruper Chaussee 149, 22761 Hamburg, Germany \\ ${ }^{4}$ Physikalisches Institut, Universität Freiburg, Hermann-Herder-Strasse 3, 79104 Freiburg, Germany \\ ${ }^{5}$ Westfälische Hochschule, Münsterstrasse 265, 46397 Bocholt, Germany
}

(Received 8 August 2016; published 28 November 2016)

\begin{abstract}
We show that strong non-Markovian effects can be revealed by the steady-state two-dimensional (2D) photon echo spectra at asymptotic waiting times. For this, we use a simple dimer toy model that is strongly coupled to a harmonic bath with parameters typical for photoactive biomolecules. We calculate the 2D photon echo spectra employing both the numerically exact hierarchy equation of motion and the quasiadiabatic path integral approach and compare these results with approximate results from a time-nonlocal quantum master equation approach. While the latter correctly reproduces the exact population dynamics at long times, it fails at the same time to correctly describe the 2D photon echo spectra at long waiting times. The differences show that non-Markovian effects are much more important for the steady-state 2D photon echoes than for the equilibrium populations. Thus, accurate theoretical descriptions of the energy transfer dynamics in biomolecular complexes have to be based on numerically exact simulations of the environmental fluctuations when nonlinear response functions are analyzed.
\end{abstract}

DOI: 10.1103/PhysRevE.94.052146

\section{INTRODUCTION}

In recent years, ultrafast nonlinear optical spectroscopy has been developed into a very successful tool to study the nonequilibrium dynamics of quantum systems [1-3]. For example, it allows us to probe and analyze highly complex artificial photoactive and natural photosynthetic complexes on the femtosecond time scale revealing their excitonic energy transfer dynamics [4-11].

Theoretical descriptions commonly employ a Frenkelexciton model within a system-bath approach. Thereby, one focuses on the excitonic degrees of freedom and few vibrational modes strongly coupled to the exciton system. All other electronic and vibrational degrees of freedom are treated as a harmonic bath [12], i.e., a source of environmental fluctuations which are finally integrated out in a statistical average. Often the strong environmental fluctuations acting in photosynthetic complexes at ambient temperatures prohibit perturbative treatments of the system-bath coupling $[13,14]$ and ask for numerically exact procedures. Usually, however, for complexes with many relevant degrees of freedom only approximate treatments of the system-bath coupling are numerically efficient [7-9]. For small photosynthetic complexes, like the Fenna-Mathews-Olsen complex, the hierarchy equation of motion (HEOM) has successfully been implemented $[15,16]$ and, by way of massive parallelization, even a study of the LH1 + LH2 complex of the purple photosynthetic bacteria became possible, which involves a 50-pigment system [17]. The quasiadiabatic path integral (QUAPI) approach [18-20] can also be employed and has the advantage that arbitrary spectral functions of the environment can be treated, in contrast to the HEOM approach. However, the numerical effort is immense and only a dimer system can be efficiently tackled by QUAPI when also the double excited states are to be taken into account.
A particular form of nonlinear spectroscopy is twodimensional photon echo spectroscopy in which three laser pulses with wave vectors $\vec{k}_{1}, \vec{k}_{2}$, and $\vec{k}_{3}$ act on the complex and, then, the out-coming signal in $\vec{k}_{s}=-\vec{k}_{1}+\vec{k}_{2}+\vec{k}_{3}$ is recorded. When the employed laser pulses are very short compared to any time scale of the dynamics of interest, they can be treated as $\delta$ pulses, which then allows us to make use of nonlinear response functions [1-3]. Typical pulses last for tens of femtoseconds, which is similar to typical dephasing and excitonic oscillation times. Then, the full extended time range of the pulses has to be taken into account. Subsequently, only the time evolution of the total dipole operator can be determined. Various approaches then allow us to determine the 2D photon echo signal. For instance, the high-order polarization can be calculated by the perturbative expansion of the light-matter interactions and the photon echo can be obtained by the appropriate combination of the selected polarization signals [21]. Moreover, the photon echo signal can be calculated by the phase-matching approach, which disentangle the signal into certain phase-matching directions [22,23].

Non-Markovian effects emerging at strong system-bath coupling [13] and/or small maximal bath frequency [14] are well established in the study of the population dynamics and the coherent (short time) excitonic dynamics observed in 2D photon echoes for rather short waiting times, i.e., $T \lesssim 200$ fs. Energy transfer in photosynthetic complexes occurs on longer time scales, i.e., $\sim 1000$ fs. Here, the typical transfer dynamics is mainly incoherent and strong system-bath influences are believed to result mainly in renormalizing the equilibrium populations of the excitonic states. Thus, biomolecular energy transfer in large photosynthetic complexes is typically modeled by employing numerically inexpensive but approximate methods. While such approximate approaches are often accepted to be reliable for the investigation of the mere population dynamics, their reliability in connection with advanced 
and refined nonlinear spectroscopic techniques is usually not considered. This is the starting point of the present work.

In this paper, we show that the steady-state $2 \mathrm{D}$ photon echo spectra calculated with different approaches may differ significantly, although, for the same parameters, the equilibrium populations do not differ. To illustrate this, we compare numerically exact calculations with approximate calculations. This shows that non-Markovian effects play an important role in 2D photon echoes at long waiting times. Conversely, $2 \mathrm{D}$ photon echoes can be used to reveal these effects in photoactive complexes. In detail, we show this effect by employing a simple dimer model strongly coupled to a bath introduced in Sec. II. We determine the 2D photon echo spectra employing the two numerically exact approaches of the hierarchy equations of motion (HEOM) and the QUAPI. We compare these results with approximate results from a time-nonlocal quantum master equation (TNL) [24]. All methods are shortly discussed in Sec. III. In Sec. IV we give our concrete results. Section V concludes the paper.

\section{DIMER MODEL}

We investigate a dimer system consisting of two monomers. For each monomer $j=1,2$, we consider a single optical excitation with ground $S_{0}\left(\left|g_{j}\right\rangle\right)$ and first excited state $S_{1}$ $\left(\left|e_{j}\right\rangle\right)$ separated by the excitation energy $E_{j}$. Additionally, each monomer is coupled via $H_{S B, j}=\left|e_{j}\right\rangle\left\langle e_{j}\right| \sum_{k} \lambda_{k}\left(b_{k, j}^{\dagger}+b_{k, j}\right)$ to its separate environment described as a harmonic bath with the Hamiltonian $H_{B, j}=\sum_{k} \omega_{k} b_{k, j}^{\dagger} b_{k, j}$. Here, $\lambda_{k}$ is the coupling constant between monomer and bath mode $k$ with annihilation/creation operator $b_{k, j}^{\dagger} / b_{k, j}$. In total, we obtain the monomer Hamiltonian

$$
H_{j}=\left|e_{j}\right\rangle\left\langle e_{j}\right|\left(E_{j}+\sum_{k} \lambda_{k}\left(b_{k, j}^{\dagger}+b_{k, j}\right)\right)+H_{B, j} .
$$

Each monomer has a transition dipole moment $\hat{\mu}_{j}=$ $\vec{\mu}_{j}\left(\left|g_{j}\right\rangle\left\langle e_{j}|+| e_{j}\right\rangle\left\langle g_{j}\right|\right)$. We assume the two dipole moments of both monomers to have the same amplitudes and to be orthogonal to each other, i.e., $\vec{\mu}_{1} \perp \vec{\mu}_{2}$. The total dipole moment $\hat{\mu}_{1}+\hat{\mu}_{2}=X+X^{\dagger}$, with

$$
X=\vec{\mu}_{1}\left|g_{1}\right\rangle\left\langle e_{1}\left|+\vec{\mu}_{2}\right| g_{2}\right\rangle\left\langle e_{2}\right|,
$$

couples to the electric field,

$$
\vec{E}_{l}(t)=\hat{e}\left[E_{l}(t) e^{-i \omega t-i \vec{k}_{l} \vec{r}}+\text { c.c. }\right]
$$

of the pulse $l$. Therein, $E_{l}(t)=\exp \left[-4 \ln 2\left(t-t_{l}\right)^{2} / \tau_{p}^{2}\right]$ denotes the pulse profile with width $\tau_{p}$, which peaks at $t_{l}, \vec{k}_{l}$ the wave vector, $\hat{e}$ its polarization vector, and $\omega$ the respective frequency of the laser pulse and $\vec{r}$ the position of the dimer in space.

The dipolar coupling between the dipole moments of the monomers results in a dimer with Hamiltonian

$$
H_{d}=H_{1}+H_{2}+J\left(\left|g_{1} e_{2}\right\rangle\left\langle e_{1} g_{2}|+| e_{1} g_{2}\right\rangle\left\langle g_{1} e_{2}\right|\right),
$$

with dipolar coupling constant $J$.

\section{A. Choice of model parameter}

We study a system with coupling $J=150 \mathrm{~cm}^{-1}$. Optical excitation energies of a chromophore monomer are considered to be in the optical regime, i.e., $E_{j} \sim 15000 \mathrm{~cm}^{-1}$, with an energy difference $E_{2}-E_{1}=100 \mathrm{~cm}^{-1}$. The laser frequency $\omega_{0}$ will be centered in between both exciton energies and we apply the rotating wave approximation so that we might set the site energies reduced by $\omega_{0}$ leading to $E_{2}=50 \mathrm{~cm}^{-1}$ and $E_{1}=-50 \mathrm{~cm}^{-1}$. We fix the laser pulse length to $\tau_{p}=40 \mathrm{fs}$.

We assume that the bath coupled to each monomer is described by the same Ohmic spectral function $J(\omega)=$ $\pi \sum_{k} \lambda_{k}^{2} \delta\left(\omega-\omega_{k}\right)=\gamma \omega f\left(\omega, \omega_{c}\right)$ with a dimensionless coupling strength $\gamma$ and a cutoff function $f\left(\omega, \omega_{c}\right)$ with cutoff frequency $\omega_{c}$. We compare results obtained for a Debyelike cutoff function $f_{D}\left(\omega, \omega_{c}\right)=\omega_{c}^{2} /\left(\omega_{c}^{2}+\omega^{2}\right)$ with results for an exponential cutoff function $f_{e}\left(\omega, \omega_{c}\right)=\exp \left(-\omega / \omega_{c}\right)$. We assume both baths to have no cross correlations, i.e., $\left\langle b_{k, 1}^{\dagger}(t) b_{k, 2}\left(t_{0}\right)\right\rangle=0$. We consider a strong system-bath coupling with $\gamma=1$ and $\omega_{c}=150 \mathrm{~cm}^{-1}$ as typical in biomolecular systems [25].

The reorganization energy $E_{R}=\frac{1}{\pi} \int_{0}^{\infty} d \omega J(\omega) / \omega$ can be evaluated to be $\sim 48 \mathrm{~cm}^{-1}$ for the exponential cutoff and $75 \mathrm{~cm}^{-1}$ in the case of the Debye like cutoff function. Furthermore, we assume the bath being initially in thermal equilibrium at the temperature $T_{0}=300 \mathrm{~K}$ resulting in an initial state given by the equilibrium density operator $\rho(0)=$ $\left|g_{1}\right\rangle\left\langle g_{1}|\otimes| g_{2}\right\rangle\left\langle g_{2}\right| \otimes \rho_{B}^{(e q)}$ with $\rho_{B}^{(e q)}=e^{-\left(H_{B, 1}+H_{B, 2}\right) / T_{0}} / Z_{\mathrm{ph}}$ and $Z_{\mathrm{ph}}=\operatorname{Tr}\left[e^{-\left(H_{B, 1}+H_{B, 2}\right) / T_{0}}\right]$ with $k_{B}=1$.

To simulate experimentally realistic situations, we take inhomogeneous broadening into account by a diagonal energetic static disorder characterized by a Gaussian distribution with the width $\sigma=50 \mathrm{~cm}^{-1}$ (FWHM). We convolute the calculated homogeneous spectra with the Gaussian-shaped inhomogeneous broadening function. Furthermore, we consider an all-parallel setup for the three laser pulse polarizations and an isotropic distribution of fixed molecules. We orientationally average our results accordingly.

\section{METHODS}

To determine the 2D photon echo response of the model dimer to the three incident laser pulses we employ a phase matching approach perturbative in the light-matter interaction [22]. The underlying open quantum dynamics is calculated by the approximate TNL quantum master equation, by the HEOM approach and by the numerically exact QUAPI approach. Below, we briefly summarize these well-established techniques.

\section{A. 2D photon echoes for finite-length laser pulses}

In the perturbative phase-matching approach [22], the polarization of the dimer in the photon-echo direction is calculated by simultaneously propagating three auxiliary density matrices $\rho_{1}, \rho_{2}$, and $\rho_{3}$. Employing the rotating-wave approximation leads to the auxiliary dynamical equations

$$
\begin{aligned}
& \partial_{t} \rho_{1}(t)=-i\left[H_{d}-V_{1}(t)-V_{2}^{\dagger}(t)-V_{3}^{\dagger}(t), \rho_{1}(t)\right], \\
& \partial_{t} \rho_{2}(t)=-i\left[H_{d}-V_{1}(t)-V_{2}^{\dagger}(t), \rho_{2}(t)\right], \\
& \partial_{t} \rho_{3}(t)=-i\left[H_{d}-V_{1}(t)-V_{3}^{\dagger}(t), \rho_{3}(t)\right],
\end{aligned}
$$


where $V_{j}(t)=X E_{j}(t)$. To obtain the third-order 2D photon echo signal, the polarization in the phase matching direction is

$$
P_{\mathrm{PE}}\left(t_{1}, t_{2}, t_{3}\right)=\left\langle X\left(\rho_{1}(t)-\rho_{2}(t)-\rho_{3}(t)\right)\right\rangle+\text { c.c. },
$$

where the bracket $\langle\ldots\rangle$ denotes the trace and the times $t_{j}$ refer to the peak times in the respective pulse profiles. Experimentally, in the limit of ideal detection, the heterodyne photon echo signal is proportional to the polarization $P_{\mathrm{PE}}\left(t_{1}, t_{2}, t_{3}, t\right)$, where $t$ is the detection time. Therefore, the ideal total 2D signal can be expressed as

$$
S_{T}\left(\omega_{\tau}, T, \omega_{t}\right) \propto \int_{-\infty}^{\infty} d \tau \int_{-\infty}^{\infty} d t e^{-i \omega_{\tau} \tau+i \omega_{t} t} i P_{\mathrm{PE}}(\tau, T, t),
$$

where $\tau, T$, and $t$ denote the coherence time, the population (waiting) time, and the detection time, respectively, $\tau=t_{2}-t_{1}, T=t_{3}-t_{2}$. We note that all the pulses are assumed to have the same line shape, carrier frequencies, and durations.

\section{B. Time nonlocal quantum master equation}

The dynamic Eqs. (3) can be solved employing established techniques of open quantum systems. A numerically highly efficient choice is the time-nonlocal quantum master equation (TNL),

$$
\dot{\rho}_{s}(t)=\mathcal{L}_{s}^{\mathrm{eff}} \rho_{s}(t)+\int_{0}^{t} K\left(t, t^{\prime}\right) \rho_{s}\left(t^{\prime}\right)+\Gamma(t),
$$

which is based on the Zwanzig equation of motion with a projection scheme. The time-evolution of the reduced system density operator $\rho_{s}(t)$ is described by a system part $\mathcal{L}_{s}^{\text {eff }} \rho_{s}(t)$, a memory term $\int_{0}^{t} K\left(t, t^{\prime}\right) \rho_{s}\left(t^{\prime}\right)$, and the inhomogeneous term $\Gamma(t)$. The latter is associated to potential initial correlations between system and bath. Based on the second-order approximation of the system-bath interactions, the use of the memory kernel $K\left(t, t^{\prime}\right)$ can be avoided by introducing two types of auxiliary density matrices $\rho^{r}(t), \rho^{i}(t)$. The time-retarded Eq. (6) is then elegantly deconvoluted into coupled first-order ordinary differential equations, which then can be solved numerically very efficiently. More details can be found in Ref. [24].

\section{Hierarchy equation of motion}

The derivation of the hierarchy equation of motion (HEOM) can be found in Ref. [26]. For a spectral density with Dude cutoff, the bath autocorrelation function can be expressed as an exponential in the form $c(t)=2 E_{R} \exp \left(-\omega_{c} t\right)$. Here, $E_{R}$ and $\omega_{c}$ is the reorganization energy and the cutoff frequency, respectively. Within a high-temperature approximation $\beta \hbar \gamma_{j}<1$, the HEOM follows as

$$
\begin{aligned}
\dot{\rho}_{s}(t)= & -\left(i \mathcal{L}+\sum_{j=1}^{N} n_{j} \gamma_{j}\right) \rho(n, t) \\
& +\sum_{j=1}^{N}\left[\Phi_{j} \rho_{s}\left(n_{j+}, t\right)+n_{j} \Theta_{j} \rho_{s}\left(n_{j-}, t\right)\right],
\end{aligned}
$$

for a set of nonnegative integers, $n=\left(n_{1}, n_{2}, \ldots, n_{N}\right) . n_{j \pm}$ is the hierarchy note by changing from $n_{j}$ to $n_{j} \pm 1$. The relaxation operators are given by $\Phi_{j}=i\left(\left|e_{j}\right\rangle\left\langle e_{j}\right|\right)^{x}$, $\Theta_{j}=i\left(\frac{2 E_{R}}{\beta \hbar^{2}}\left(\left|e_{j}\right\rangle\left\langle e_{j}\right|\right)^{x}-i \frac{E_{R}}{\hbar} \omega_{c}\left(\left|e_{j}\right\rangle\left\langle e_{j}\right|\right)^{\circ}\right)$, where the operator $O^{x} f=O f-f O$ and $O^{\circ} f=O f+f O$.

\section{Quasiadiabatic propagator path-integral}

To calculate the spectra with the exponential cutoff function numerically exactly, the QUAPI $[18,19]$ is typically more efficient than the HEOM. In brief, the algorithm is based on a symmetric Trotter splitting of the short-time propagator for the full Hamiltonian into two parts, one depending on the system Hamiltonian and one involving the bath and the coupling term. The short-time propagator determines the time evolution over a Trotter time slice $\delta t$. The discrete time evolution becomes exact in the limit $\delta t \rightarrow 0$. On the other side, the environmental degrees of freedom generate correlations that are nonlocal in time. For any finite temperature, these correlations decay on a time scale denoted as the memory time scale. The QUAPI scheme defines an "augmented" reduced density tensor, which lives on this memory time window. Then, an iteration scheme is established in order to extract the time evolution of this object. All correlations are completely included over the finite memory time $\tau_{\text {mem }}=K \delta t$ but are neglected for times beyond $\tau_{\text {mem }}$. One increases the memory parameter $K$ until convergence is found, at the same time respecting the limit $\delta t \rightarrow 0$.

\section{RESULTS}

Equipped with the different tools to evaluate the quantum dissipative dynamics, we next determine the population dynamics of the dimer and the $2 \mathrm{D}$ spectra.

\section{A. Population dynamics}

We determine the population dynamics of the dimer for a Debye-like cutoff function employing the TNL and HEOM. Thereby, we start from an initially excited monomer 1 and

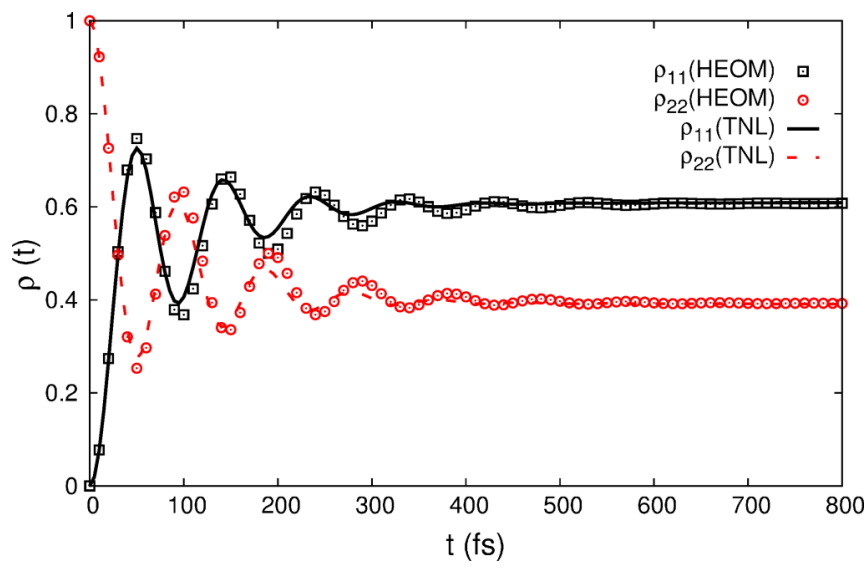

FIG. 1. Time-dependent probabilities of an excitation to be in monomer 1 or 2 , i.e., $\rho_{11}(t)$ and $\rho_{22}(t)$, for the parameters given in the text and calculated by the TNL quantum master equation and by the HEOM technique. 


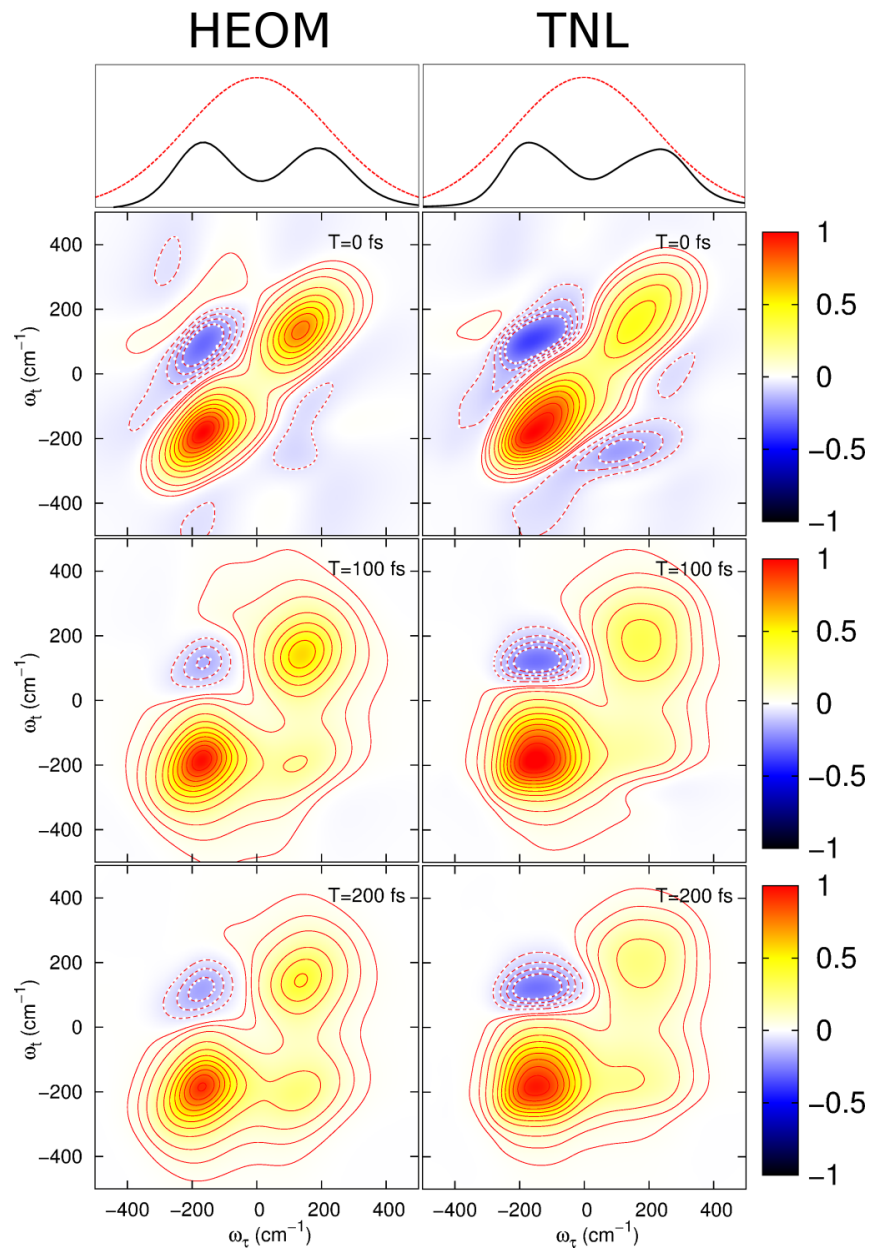

FIG. 2. Real part of the two-dimensional photon echo spectra (rephrasing and nonrephasing) for a dimer calculated for three different waiting times $T=0 \mathrm{fs}, T=100 \mathrm{fs}$, and $T=200 \mathrm{fs}$ (positive, solid lines; negative, dashed lines). The left column shows results obtained by numerically exact HEOM approach, while the right column shows the results obtained by the approximate TNL technique (for the parameters, see text). The transfer rate between the two exciton states was analyzed by the global fitting approach, which yields the time scale of 150 fs for both methods. We also show the frequency spectrum of the excitation laser (dashed red lines) and the linear photoabsorption spectra (solid black lines).

focus on the energy transfer dynamics within the singleexcitation subspace. In Fig. 1, we show the time dependence of the occupation probability $\rho_{11}(t)$ for the excitation in monomer 1 and $\rho_{22}(t)$ for the excitation in monomer 2. Despite the rather strong system-bath coupling, both methods show an oscillatory behavior for the first $500 \mathrm{fs}$ with similar oscillation frequencies. The TNL result slightly overestimates the decay times. Importantly, the TNL quantum master equation gives identical results for the long-time steady-state occupation of both states when compared to the numerically exact HEOM results.

\section{B. 2D spectra}

In Fig. 2, we depict the 2D photon echo spectra for the dimer for three different waiting times $T=0 \mathrm{fs}, T=100 \mathrm{fs}$,

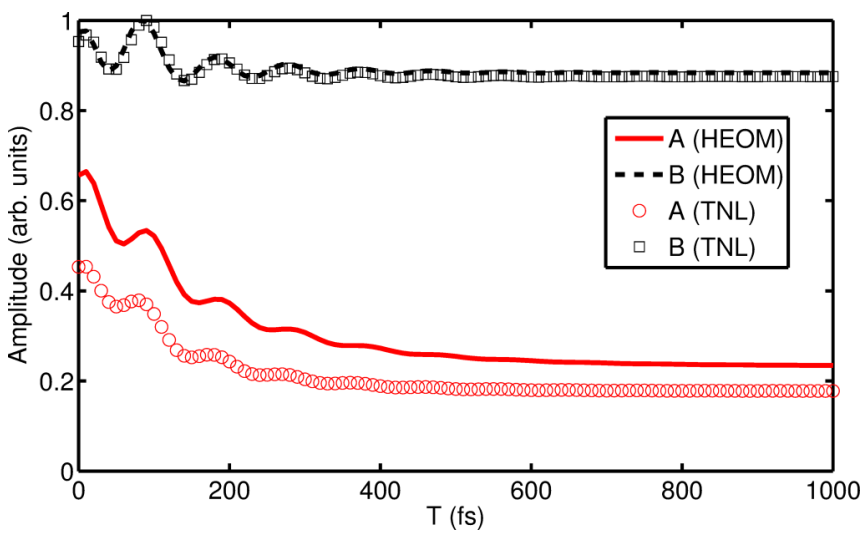

FIG. 3. Magnitude of the peaks A and B in the photon echo spectra versus waiting time $T$. The solid and dashed lines mark the HEOM results, while the markers (square and circle) indicate the TNL results.

and $T=200 \mathrm{fs}$. The left column shows the results obtained by the numerically exact HEOM approach and data plotted in the right column are calculated by the approximate TNL approach. As expected, both techniques yield qualitatively and, in big parts, also quantitatively identical results for the $2 \mathrm{D}$ photon echo spectra. Nevertheless, clear differences are also visible. The off-diagonal peak above the diagonal at $\omega_{t} \simeq 140 \mathrm{~cm}^{-1}$ and $\omega_{\tau} \simeq-140 \mathrm{~cm}^{-1}$ (denoted as peak $\mathrm{C}$ in the following) is more pronounced in the TNL calculations.

A more detailed quantitative comparison is achieved when waiting time traces at specific points in the $2 \mathrm{D}$ photon echoes are analyzed, specifically for the different peaks. In Fig. 3, the maximal height of the diagonal peak $\mathrm{A}$ (red lines), with $\omega_{t} \simeq 170 \mathrm{~cm}^{-1}$ and $\omega_{\tau} \simeq 170 \mathrm{~cm}^{-1}$, and the diagonal peak B (black lines), with $\omega_{t} \simeq-190 \mathrm{~cm}^{-1}$ and $\omega_{\tau} \simeq-190 \mathrm{~cm}^{-1}$, are plotted versus waiting time $T$ up to $1000 \mathrm{fs}$. Figure 4 shows the maximal height of the off-diagonal peak $\mathrm{C}$ (blue lines) at $\omega_{t} \simeq$ $170 \mathrm{~cm}^{-1}$ and $\omega_{\tau} \simeq-190 \mathrm{~cm}^{-1}$ and the off-diagonal peak $\mathrm{D}$ (magenta lines) at $\omega_{t} \simeq-190 \mathrm{~cm}^{-1}$ and $\omega_{\tau} \simeq 190 \mathrm{~cm}^{-1}$ accordingly. The full lines are HEOM results and the dashed lines TNL results. The results of the TNL and the numerically exact HEOM approach agree qualitatively well for all peaks. Quantitatively, the decay times and the oscillation frequencies obtained from both approaches for the short time behavior,

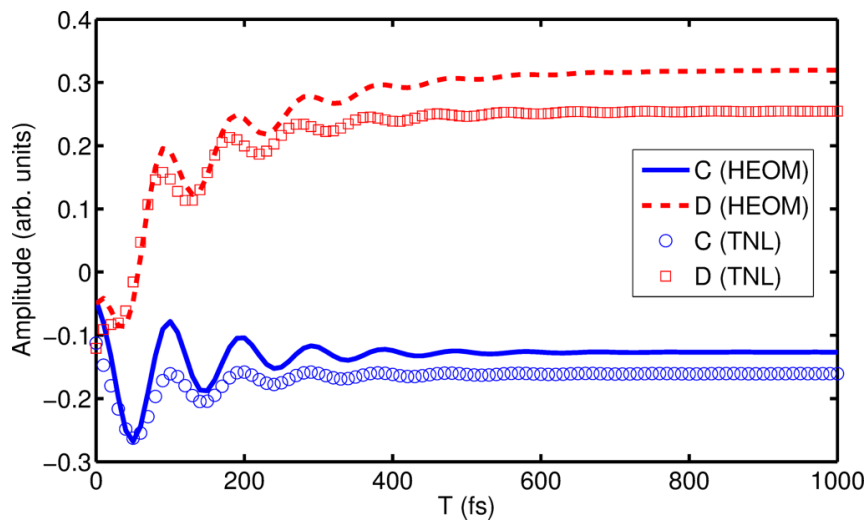

FIG. 4. Same as in Fig. 3, but for peaks C and D. 


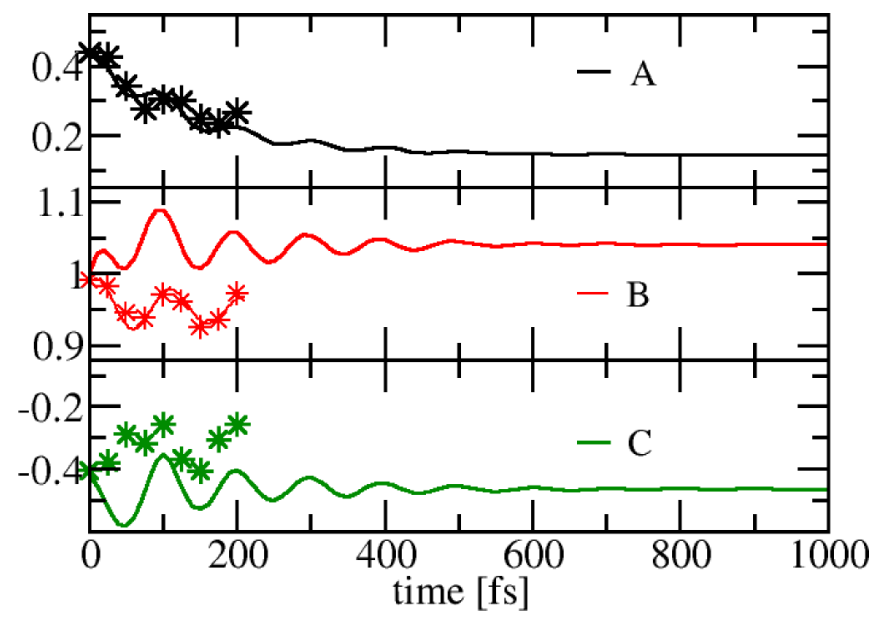

FIG. 5. Heights of the peaks A, B, and C in the photon echo spectra for an exponentially cutoff bath spectral function versus increasing waiting time. The symbols indicate the numerically exact QUAPI results and the dashed lines mark the approximate TNL results.

i.e., $T \lesssim 500$ fs, agree reasonably well. However, except for peak $\mathrm{B}$ the results for the long-time peak heights determined by the TNL approach differ clearly from the numerically exact HEOM result.

Since the TNL approach is based on the Markovian approximation, our results reveal the difference of the kinetics calculated by the non-Markovian approach although the results for the population dynamics (see Fig. 1) agree well. The 2D spectra show clear differences both in the asymptotic values and in the decay times and manifest non-Markovian effects.

\section{Exponential cutoff function}

In order to check how sensitive our results are to the high-frequency regime of the environmental fluctuational spectrum, we have additionally studied the dimer model with an exponential cutoff function instead of a Debye-like one. In this case, the HEOM technique is less efficient to treat the dissipative influence of the bath, since the HEOM relies on the exponential form of the bath autocorrelation function, which is not strictly given for the exponential cutoff. Since the QUAPI approach does not suffer from this limitation, we have used it to compare to the TNL quantum master equation. The results for the waiting time traces for the various peak heights are shown in Fig. 5. Due to the immense numerical costs of QUAPI, only a limited set of waiting times can be studied. The results are marked by the symbols in Fig. 5. The comparison of the QUAPI results with the TNL results (solid lines in Fig. 5) shows nevertheless that for peak B and $\mathrm{C}$ clearly the long-time steady-state values significantly differ. Thus, the same conclusions as for the Debye spectral function can be drawn, namely that non-Markovian effects due to strong system-bath coupling strongly influence the steady-state 2D photon echo spectra at long waiting times although their influence is relatively weak for the population dynamics of the dimer.

\section{v. CONCLUSION}

In large photosynthetic clusters, the time scale of the energy transfer is commonly determined by the weak interactions between the molecular subunits. Often, these subunits reach a steady state on the same time scale after being initially excited. Hence, strong-damping approaches are required, which properly describe the rather fast subunit relaxation, while the transfer dynamics between different subunits evolves on a comparable time scale. For theoretical studies of such large photosynthetic clusters, often only approximate methods are numerically efficient when the influence of environmental degrees of freedom is taken into account. They are regularly based on the Markovian approximation and it becomes a valid question whether weak-coupling Markovian approaches are accurate enough to properly describe such a dynamical process.

As reported in this work, this is not the case and approximate Markovian tools have to be used with care when nonlinear response functions such as $2 \mathrm{D}$ photon echo spectra are calculated. We show here that considerable non-Markovian effects due to a strong system-bath coupling prevail on long waiting times in the 2D spectra, even when such effects are not visible in the population dynamics of the same model. In particular, the steady-state $2 \mathrm{D}$ photon echo spectra differ significantly, which is illustrated by comparing numerically exact results with approximate results for a simple dimer model. It is important to realize that the differences persist although the equilibrium populations do not differ. This result is also confirmed by the work of Ref. [27]. In addition, the comparison of time-local (TL) and TNL approaches has been done by Ref. [28] and they found that, for certain cases, TL is quite superior to the TNL master equation. The benchmark study of TL and TNL methods should be done and compared to the numerically exact method in the future. This, in turn, forces one to employ numerically exact methods also when analyzing the long-time energy transfer dynamics in photosynthetic complexes. Likewise, 2D photon echo spectra can be used to reveal strong non-Markovian effects at play.

\section{ACKNOWLEDGMENTS}

We acknowledge financial support by the Joachim-HerzStiftung, Hamburg, within the PIER Fellowship program and by the excellence cluster "The Hamburg Centre for Ultrafast Imaging-Structure, Dynamics and Control of Matter at the Atomic Scale" of the Deutsche Forschungsgemeinschaft. P.N. acknowledges financial support by the Deutsche Forschungsgemeinschaft (DFG) Project No. NA394/2-1.
[1] S. Mukamel, Principles of Nonlinear Optics and Spectroscopy (Oxford University Press, New York, 1995).
[2] S. Mukamel, Annu. Rev. Phys. Chem. 51, 691 (2000). 
[3] M. Cho, Two-Dimensional Optical Spectroscopy (CRC Press, Boca Raton, 2009).

[4] T. Brixner, J. Stenger, H. Vaswani, M. Cho, R. E. Blankenship, and G. R. Fleming, Nature 434, 625 (2005).

[5] G. S. Engel, T. R. Calhoun, E. L. Read, T. K. Ahn, T. Mančal, Y.-C. Cheng, R. E. Blankenship, and G. R. Fleming, Nature 446, 782 (2007).

[6] E. Collini, C. Y. Wong, K. E. Wilk, P. M. G. Curmi, P. Brumer, and G. D. Scholes, Nature 463, 644 (2010).

[7] A. Halpin, P. J. M. Johnson, R. Tempelaar, R. S. Murphy, J. Knoester, T. L. C. Jansen, and R. J. D. Miller, Nat. Chem. 6, 196 (2014).

[8] E. Romero, R. Augulis, V. I. Novoderezhkin, M. Ferretti, J. Thieme, D. Zigmantas, and R. van Grondelle, Nature Phys. 10, 676 (2014).

[9] F. D. Fuller, J. Pan, A. Gelzinis, V. Butkus, S. S. Senlik, D. E. Wilcox, C. F. Yocum, L. Valkunas, D. Abramavicius, and J. P. Ogilvie, Nature Chem. 6, 706 (2014).

[10] V. I. Novoderezhkin, M. A. Palacios, H. van Amerongen, and R. van Grondelle, J. Phys. Chem. B 109, 10493 (2005).

[11] G. S. Schlau-Cohen, T. R. Calhoun, N. S. Ginsberg, E. L. Read, M. Ballottari, R. Bassi, R. van Grondelle, and G. R. Fleming, J. Phys. Chem. B 113, 15352 (2009).

[12] V. May and O. Kühn, Charge and Energy Transfer Dynamics in Molecular Systems (Wiley-VCH, Weinheim, 2011).
[13] A. Ishizaki and G. R. Fleming, J. Chem. Phys. 130, 234110 (2009).

[14] P. Nalbach and M. Thorwart, J. Chem. Phys. 132, 194111 (2010).

[15] L. Chen, R. Zheng, Y. Jing, and Q. Shi, J. Chem. Phys. 134, 194508 (2011).

[16] C. Kreisbeck and T. Kramer, J. Phys. Chem. Lett. 3, 2828 (2012).

[17] J. Strümpfer and K. Schulten, J. Chem. Theory Comput. 8, 2808 (2012).

[18] N. Makri and D. E. Makarov, J. Chem. Phys. 102, 4600 (1995).

[19] N. Makri and D. E. Makarov, J. Chem. Phys. 102, 4611 (1995).

[20] P. Nalbach and M. Thorwart, Phys. Rev. Lett. 103, 220401 (2009).

[21] T. Mančal, A. V. Pisliakov, and G. R. Fleming, J. Chem. Phys. 124, 234504 (2006).

[22] M. F. Gelin, D. Egorova, and W. Domcke, Chem. Phys. 312, 135 (2005).

[23] P. Kjellberg, B. Brüggemann, and T. Pullerits, Phys. Rev. B 74, 024303 (2006).

[24] C. Meier and D. Tannor, J. Chem. Phys. 111, 3365 (1999).

[25] Y.-C. Cheng and G. R. Fleming, J. Phys. Chem. A 112, 4254 (2008).

[26] A. Ishizaki and Y. Tanimura, J. Phys. Soc. Japan 74, 3131 (2005).

[27] L. Chen, R. Zheng, Q. Shi, and Y. J. Yan, J. Chem. Phys. 132, 024505 (2010).

[28] M. Schröder, M. Schreiber, and U. Kleinekathöfer, J. Chem. Phys. 126, 114102 (2007). 